\title{
An open-label, non-randomized comparison of venlafaxine and gabapentin as monotherapy or adjuvant therapy in the management of neuropathic pain in patients with peripheral neuropathy
}

This article was published in the following Dove Press journal: Journal of Pain Research

31 March 2010

Number of times this article has been viewed

\section{William Eardley \\ Cory Toth}

Department of Clinical Neurosciences and the University of Calgary, Calgary, $A B$, Canada
Correspondence: Cory Toth HMRB Room I55, University of Calgary, Department of Clinical Neurosciences, 3330 Hospital Drive NW, Calgary, Alberta, Canada T2N 4NI

$\mathrm{Tel}+\mathrm{I}(403)$ 220-883 I

Fax + I (403) 283-873I

Email corytoth@shaw.ca
Abstract: Although many therapies are used in the management of neuropathic pain (NeP) due to polyneuropathy $(\mathrm{PN})$, few comparison studies exist. We performed a prospective, non-randomized, unblended, efficacy comparison of the serotonin-norepinephrine reuptake inhibitor venlafaxine, as either monotherapy or adjuvant therapy, with a first-line medication for NeP, gabapentin, in patients with PN-related NeP. VAS pain scores were assessed after 3 and 6 months in intervention groups and in a cohort of patients receiving no pharmacotherapy. In a total of 223 patients, we analyzed pain quantity and quality (visual analogue scale [VAS] score, Brief Pain Inventory [BPI]), quality of life and health status measures [EuroQol 5 Domains, EQ-5D], Medical Outcomes Sleep Study Scale [MOSSS], Hospital Anxiety and Depression Scale [HADS] and Short Form 36 Health Survey [SF-36]) after 6 months of therapy. Significant improvements in VAS pain scores occurred for all treatment groups after 6 months. Improvements in aspects of daily life and anxiety were identified in all treatment groups. Our data suggest that monotherapy or adjuvant therapy with venlafaxine is comparable to gabapentin for $\mathrm{NeP}$ management. We advocate for head-to-head, randomized, double-blinded studies of current NeP therapies.

Keywords: peripheral neuropathy, neuropathic pain, pharmacotherapy, venlafaxine, gabapentin

\section{Introduction}

Chronic polyneuropathy (PN) is a common condition ${ }^{1}$ estimated to occur in about $2.4 \%$ of the population, increasing to $8 \%$ in the elderly. ${ }^{2}$ Etiologies of $\mathrm{PN}$ are numerous, including diabetes mellitus, vitamin B12 deficiency, alcohol, vasculitis and immunerelated diseases ${ }^{1}$ as well as idiopathic causes. ${ }^{3}$ Neuropathic pain $(\mathrm{NeP})$, referring to pain of peripheral or central nervous system origin and characterized by continuous or paroxysmal dysesthesias, occurs in up to $50 \%$ of patients with PN., ${ }^{4,5}$ Patients with $\mathrm{NeP}$ often describe burning, shooting or stabbing electrical sensations; allodynia and hyperalgesia may also occur. ${ }^{1}$ Chronic NeP reduces quality of life in several domains, and concomitant mood and sleep disorders often co-occur. ${ }^{6-9}$

Diversity in the management of NeP related to variations in severity, concomitant syndromes, and patient diversity, make NeP particularly challenging to manage. ${ }^{10,11}$ Further, side effects often limit the beneficial effects of $\mathrm{NeP}$ pharmacotherapy. ${ }^{12}$ 
Even with use of a first-line agent such as gabapentin for $\mathrm{NeP},{ }^{13}$ only $67 \%$ of treated patients are able to tolerate a maximal therapeutic dosage and most of these patients still tolerate dizziness and somnolence. ${ }^{5}$ Despite the presence of guidelines for multiple pharmacotherapies for $\mathrm{NeP},{ }^{13,14}$ there are very few head-to-head studies to guide the clinician in making therapeutic decisions. ${ }^{15}$

Gabapentin, a 3-alkylated analogue of $\gamma$-amino butyric acid, is a well established anticonvulsant in the management of NeP. ${ }^{16-18}$ Gabapentin's predominant activity in the relief of $\mathrm{NeP}$ is the modulation of calcium channels through its binding to $\alpha_{2}-\delta$-subunit of the calcium channel complex, ${ }^{12}$ reducing pre-synaptic release of neurotransmitters. Gabapentin does not act upon serotonin or norephinephrine reuptake, ${ }^{19}$ so its mechanisms of action are distinctly different from agents such as the serotonin-norepinephrine reuptake inhibitors (SNRIs). In Canada, gabapentin is considered first-line therapy for $\mathrm{NeP}$ management ${ }^{13}$ and therefore a reasonable comparator for other agents used for $\mathrm{NeP}$.

Newer management options include venlafaxine, one of the first-used SNRIs. ${ }^{20-25}$ Beyond reuptake inhibition of serotonin and norepinephrine, venlafaxine also possesses sodium channel blockade activity, as seen with tricyclic antidepressant (TCA) medications,${ }^{24}$ weak dopamine reuptake inhibitor activity, and mild NMDA antagonism activity. ${ }^{26}$ Although venlafaxine may be associated with rare cardiac arrhythmias, suggesting the need for ongoing cardiac monitoring, ${ }^{14}$ its side effect profile is still preferable to those associated with TCAs, first-line agents for management of NeP. ${ }^{13}$ It is hypothesized that beneficial effects of SNRIs in $\mathrm{NeP}$ are seen only at higher doses at which the metabolite $\mathrm{R}$-O-desmethylvenlafaxine inhibits noradrenaline; ${ }^{27}$ therefore, it is believed that dosing of venlafaxine below $150 \mathrm{mg}$ daily is ineffective for NeP. Further research into the benefits of venlafaxine for management of $\mathrm{NeP}$ remains necessary.

Although some studies have identified potential benefits of venlafaxine and gabapentin combination therapy, ${ }^{26}$ there are no studies that have compared the two therapies headto-head. The aim of this study was to compare the relative efficacy of these two contrasting therapies in the management of $\mathrm{NeP}$ in a real-life clinical setting. A specific emphasis was placed on analyzing reductions in pain perception and changes to other pain-related symptom scales and quality of life indices. We hypothesized that the two treatments groups would have unique efficacies in the treatment of $\mathrm{NeP}$ and that both groups would fare better than a control (no treatment) group.

\section{Materials and methods}

\section{Patient assessment}

We prospectively evaluated patients with PN-related NeP in a tertiary care neuromuscular clinic in Calgary. While patients were identified prospectively, this investigation was not designed as a randomized study or a prospective cohort examination, but rather as a part of their regular clinical care. All patients enrolled within these clinics provide informed consent for ethically approved assessment of their clinical outcomes during all management, studies examining their general well being at regular follow-up visits, and completion of questionnaires conducted at these clinics (Centre for Advancement of Health, University of Calgary). There was no specific consent obtained for use of gabapentin or venlafaxine - prescription of these therapies was considered a part of standard medical care. Patients with $\mathrm{PN}$-associated $\mathrm{NeP}$ were asked, "Do you have pain or discomfort over your feet and legs on a near-daily basis for more than 6 months?" All patients who responded positively with a clinical picture consistent with PN and presence of peripheral neuropathy were deemed to have $\mathrm{NeP}$ as a complicating feature of their PN. The DN4 questionnaire (DN4 questionnaire), with good sensitivity (83\%) and specificity (90\%), ${ }^{11}$ was used to identify clinical likelihood of NeP presence - only those patients with a score of $\geq 4$ were considered eligible.

Peripheral neuropathy severity was assessed using the Toronto Clinical neuropathy score (TCNS), which is based upon history and examination and emphasizes sensory deficits as compared to other measures of peripheral neuropathy severity. ${ }^{28,29}$ Initially developed for use as a simple screening tool for diabetic peripheral neuropathy, the TCNS is based upon history and examination and emphasizes sensory deficits as compared to other measurements of the severity of peripheral neuropathy. The TCNS is a validated method of evaluation for peripheral neuropathy, higher TCNS scores being positively associated with greater pathological abnormality of sural nerve fiber density ${ }^{28}$ During the assessment of their peripheral neuropathy, the most likely etiology of the neuropathy was also determined based upon laboratory investigations and clinical information. These investigations included complete blood count, electrolytes, urea, creatinine, alanine aminotransferase (ALT), aspartate aminotransferase (AST), gammaglutamyltranspeptidase (GGT), alkaline phosphatase (ALP), albumin, total bilirubin, international normalized ratio (INR), thyroid-stimulating hormone, fasting glucose, hemoglobin $A_{1 c}$, cobalamin, erythrocyte sedimentation rate, antinuclear antibody, extracted nuclear antibody testing, serum protein 
electrophoresis, rheumatoid factor, vitamin B12 levels, fasting methylmalonic acid, and fasting homocysteine levels. Other testing was completed if a specific cause was suspected. All patients had electrophysiological testing as part of their regular care. In rare situations, a peripheral nerve biopsy was performed to supplement clinical diagnosis. There is no gold standard for the diagnosis of PN, ${ }^{30,31}$ so the final clinical diagnosis was based upon the judgment of the attending neurologist. However, PN was defined to be present if a patient had a TCNS score of $\geq 3$ including the mandatory presence of sensory abnormalities on distal bilateral leg examination unless another reason for the observed findings other than PN could be inferred. Thus, patients without evidence of physical examination signs of $\mathrm{PN}$ were excluded from further assessment. Patients were excluded from further consideration if another condition other than PN such as a rheumatological disorder or peripheral vascular disease was present in the lower extremities. Peripheral vascular pulses were palpated in all cases, and if difficult to detect or absent, then patients were excluded due to possible confounding peripheral vascular disease. Likewise, patients with symptoms of pain only present during exertion were excluded due to the possibility of confounding peripheral vascular disease.

\section{Study design}

Although these patients were identified in prospective manner, this study was designed to be a prospective cohort investigation providing a study of best clinical care. No randomization or blinding was performed. Patients were seen on three occasions during the study as part of standard care. During the first visit, determination of the cause of PN, determination of the presence of $\mathrm{NeP}$, and the decision to initiate pharmacotherapy for NeP occurred. The decision to begin a specific pharmacotherapy was made by the patient after sufficient discussion of the agents and the possible anticipated adverse events in concert with the neurologist - attempts to emphasize the use of any one of the open-label medications were avoided. Attempted prior therapy for NeP in the past or present was permitted. Patients taking no medications for pain in the prior 30 days before assessment and initiating therapy were considered as receiving monotherapy. Patients taking other medications for pain relief at the time of initial visit were considered as receiving adjuvant therapy. Enrollment was not permitted if patients discontinued or modified their medications used in the 30 days prior to study initiation; these patients were asked to return after 30 days from last pain medication modification occurred, at which time their previous medication use was considered stable. In addition to monitoring those patients receiving pharmacotherapy, patients were also monitored when no pharmacotherapy was desired or selected (control group).

\section{Pharmacotherapy: venlafaxine or gabapentin monotherapy or adjuvant treatment}

Following their assessment, patients were offered pharmacotherapy as part of their pain-management protocol. Careful recording of their current $\mathrm{NeP}$ medications prior to initiation of therapy were performed. Patients were initiated on venlafaxine or gabapentin as monotherapy or adjuvant therapy. Flexible dosing was used for all patients, with varying initiation doses for venlafaxine and gabapentin; in all cases, however, medication doses were uptitrated slowly. In addition to monitoring those patients receiving pharmacotherapy, patients receiving no pharmacotherapy were also monitored (control group).

All patients were contacted via telephone 1 week after starting monotherapy or adjuvant therapy to assess for any adverse effects. Additional clinical follow-ups occurred at 3- and 6-month intervals to perform studies related to the primary and secondary objectives, when adverse events were also recorded. If tolerating the medication well at the 3 month follow-up point, patients were given the opportunity to modulate the dose either higher or lower for the remaining 3 months. Patients with benefits but with tolerable adverse events were permitted to reduce the dose of the medication being used. We attempted to ensure that concomitant pain medications were not altered in the adjuvant therapy group. Patients were advised to contact the prescribing clinic for any possible adverse effects or clinical difficulties with pain during the time course of the study.

\section{Primary outcome measure}

Data for primary outcome measures were collected in groups of patients receiving interventions as well as in control group patients. At each visit the primary outcome measure; the degree of $\mathrm{NeP}$ (quantity and quality) was evaluated using a VAS provided by a line bisection score with an unmarked $10 \mathrm{~cm}$ line between anchors of no pain on the left $(0)$ and worst possible pain on the right (10). The marked score was asked to reflect the patient's average PN-related NeP severity over the past 24 hours. The VAS was scored by line measurement in each case. The last available data points were used for calculation of VAS in the case of drop out from the study. Data for pain quantity were collected at 0-, 3- and 6-month intervals for all patients. 


\section{Secondary outcome measures}

Data for secondary outcome measures were collected in groups of patients receiving interventions as well as in control group patients. Secondary outcomes consisted of health status and quality of life assessments. The Brief Pain Inventory (BPI), EuroQol 5 Domains (EQ-5D), Medical Outcomes Sleep Study Scale (MOSSS), Hospital Anxiety and Depression Scale (HADS) and Short Form 36 Health Survey (SF-36) were determined at study entry and after 6 months. The BPI provides information on the intensity of pain as well as the degree to which pain interferes with function, and enquires about pain relief, pain quality, and the patient's perception of the cause of pain. The EQ-5D has two sections - the first section examines the health state in 5 dimensions: mobility, self-care, usual activities, pain/complaints, and anxiety/depression. We calculated EQ-5D utility scores and VAS scores as described previously. ${ }^{32,33}$ The MOSSS is a 12-item self-report sleep measure, that can be used to assess important aspects of sleep perceived by adults. ${ }^{34}$ The HADS is another self-assessment scale that has been found to be a reliable instrument for detecting states of depression and anxiety in the setting of an outpatient clinic. Its subscales are also valid measures of severity of the emotional disorder. ${ }^{35}$ The SF-36 Health Survey is a 36-item generic measure of health status. ${ }^{9}$

Medication adverse effects were documented during the telephone interview at one week after medication initiation, as well as at 3- and 6-month study visits.

To gauge global improvement, the Patient Global Impression of Change scale (PGIC) was administered at the 6-month endpoint visit. Both scales were analyzed using modified ridit transformation with the Cochran-Mantel-Haenszel procedure, with adjustment for center.

\section{Tolerability and adverse events}

An adverse event was defined as any noxious, unintended or unexpected response suspected to have a causal relationship with the medication used. Identification of intolerable side effects at the time of follow-up visit or leading to discontinuation of medication prior to the follow-up visit was determined at the multiple follow-up points. A serious adverse event was defined as any life-threatening reaction to medication requiring hospitalization, additional urgent physician assessment, or resulted in persistent or significant disability. Patients were also asked to identify any tolerable side effects felt to be related to the medication. Side effects were accumulative throughout the study, such that a side effect experienced in the first 3 months but not the next
3 months was still recorded as a side effect for both 3- and 6-month follow-ups.

\section{Data analysis}

All patients enrolled in the NeP clinic provided informed consent to have their longitudinal data analyzed. All data were analyzed using unmatched ANOVA testing between intervention groups and between time points. Data were separated to analyze patients receiving monotherapy and adjuvant therapy. The baseline pain VAS score was used for comparison to later pain scores, and the 3-month pain VAS score was compared to the 6-month VAS pain score also. Changes in pain scores during the study period were compared between treatment groups and the control cohort. An intention to treat analysis was performed once patients were seen for follow-up, with the last observations carried forward in the case of lost follow-up or discontinuation. The Cochran-Mantel-Haenszel procedure was used to analyze PGIC data. Missing data were treated using the last observation carried forward in all cases.

\section{Results}

A total of $95 \mathrm{NeP}$ patients initiated monotherapy: 43 with venlafaxine and 52 with gabapentin. A total of 109 patients were already on $\mathrm{NeP}$ pharmacotherapy and initiated on adjuvant therapy: 45 with venlafaxine and 64 with gabapentin. A cohort group of 29 patients with PN-related NeP did not receive pharmacological treatment and were monitored in an identical fashion for the 6-month follow-up period and considered a control group (Figure 1).

Patients in each treatment group and in the control group were similar with respect to age, sex, and severity of the neuropathy prior to the study initiation (Table 1). Control patients, however, had significantly lower VAS scores at baseline than each of the treatment groups. No significant difference in baseline VAS scores within treatment groups was present (ANOVA, $P=0.32$ for monotherapy groups, $P=0.44$ for adjuvant therapy groups).

After initial titration periods, venlafaxine and gabapentin dosing varied between individual patients (Table 2), but was slightly higher for each therapy in monotherapy treated patients as compared to adjuvant therapy patients (Tables 2,3). In monotherapy patients, the mean dose of venlafaxine was just over $220 \mathrm{mg}$ daily after 3 and 6 months. In patients receiving monotherapy gabapentin, the mean dose was just under $2400 \mathrm{mg}$ daily after 3 and 6 months. In adjuvant therapy patients, the mean dose of venlafaxine was just under $220 \mathrm{mg}$ daily after both 3 and 6 months. In patients 


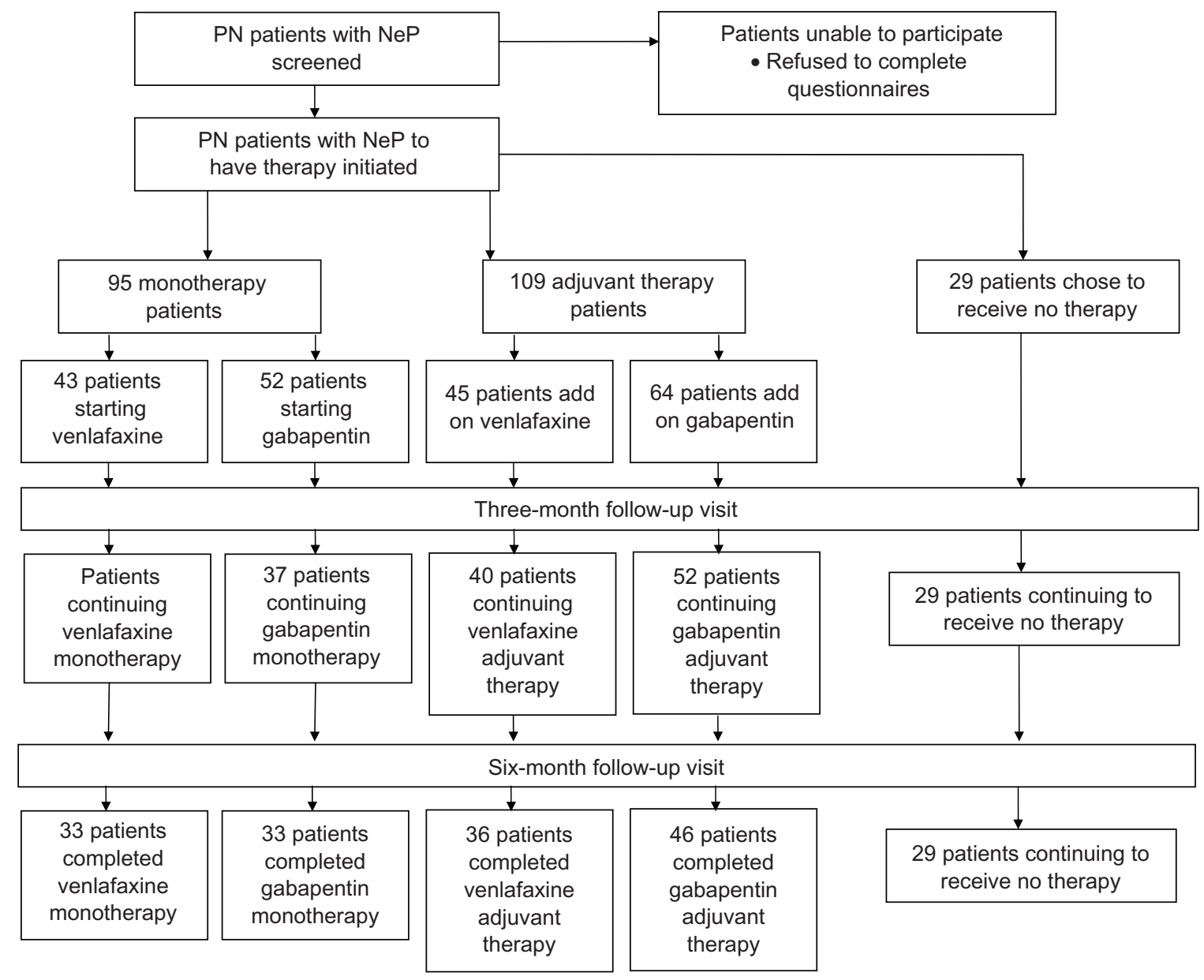

Figure I Summary of patient flow throughout study.

Abbreviations: NeP, neuropathic pain; PN, polyneuropathy.

receiving adjuvant gabapentin, the mean dose was just under $1900 \mathrm{mg}$ daily after 3 and 6 months (Tables 2, 3).

The control group data is presented in Table 4. Control group patients had less significant pain at baseline - this may have contributed to their selection not to receive pharmacotherapy. The control group also had better sleep and functioning parameter results than seen in the intervention groups.

\section{Primary outcome measures Monotherapy}

For patients treated with venlafaxine or gabapentin as monotherapy, there was a significant improvement in VAS pain scores after 3 and 6 months of treatment compared to baseline VAS pain scores. There was also a significant improvement in scores at 6 months versus 3 months for both venlafaxine and gabapentin treatment groups (Table 2). Both treatment groups had greater relative improvement in VAS pain scores when compared to control patients at 3-and 6-month follow-up visits.

\section{Adjuvant therapy}

VAS pain scores significantly improved for patients treated with venlafaxine adjuvant therapy at both 3 and 6 months compared to baseline VAS pain scores (Table 3). Venlafaxine adjuvant therapy was also associated with a significant improvement in VAS pain scores at 6-month visits versus 3-month visits. All patients treated with adjuvant therapy had greater relative improvement in VAS scores compared to control patients over the same periods of time.

\section{Secondary measures Monotherapy}

There were no significant improvements in EQ-5D scores, EQ-5D domains or EQ-Health status scores at 6-month visits versus baseline for any monotherapy treatment group (Table 2). Both gabapentin and venlafaxine monotherapy was associated with improvement in sleep disturbance and sleep adequacy within the MOSSS (Table 2). Venlafaxine monotherapy was further associated with additional improvements 
Table I Clinical features and baseline characteristics of patients and control subjects studied

\begin{tabular}{|c|c|c|c|c|c|}
\hline \multirow[t]{2}{*}{ Clinical features } & \multicolumn{2}{|c|}{ Monotherapy } & \multicolumn{3}{|l|}{ Adjuvant therapy } \\
\hline & $\begin{array}{l}\text { Venlafaxine } \\
(n=43)\end{array}$ & $\begin{array}{l}\text { Gabapentin } \\
(n=52)\end{array}$ & $\begin{array}{l}\text { Venlafaxine } \\
(n=45)\end{array}$ & $\begin{array}{l}\text { Gabapentin } \\
(n=64)\end{array}$ & $\begin{array}{l}\text { Control group } \\
\text { (no therapy) } \\
(n=29)\end{array}$ \\
\hline Age (mean \pm SD) & $59 \pm 7$ & $61 \pm 9$ & $56 \pm 5$ & $61 \pm 6$ & $62 \pm 9$ \\
\hline Female sex (\%) & 27 (63\%) & $31(60 \%)$ & $28(62 \%)$ & 37 (58\%) & 17 (59\%) \\
\hline $\begin{array}{l}\text { Age of onset of } \mathrm{NeP} \\
\text { symptoms (years), } \\
\text { mean } \pm \mathrm{SD}\end{array}$ & $57 \pm 11$ & $58 \pm 13$ & $55 \pm 9$ & $58 \pm 15$ & $59 \pm 16$ \\
\hline $\begin{array}{l}\text { Age of initiation of } \\
\text { NeP therapy } \\
\text { initiation (years), } \\
\text { mean } \pm \text { SD }\end{array}$ & $58 \pm 10$ & $59 \pm 14$ & $55 \pm 9$ & $60 \pm 14$ & $60 \pm 15$ \\
\hline \multicolumn{6}{|l|}{ Etiology of PN } \\
\hline Idiopathic & 6 & 8 & 7 & 12 & 5 \\
\hline Diabetic & 12 & 16 & 14 & 17 & 8 \\
\hline Vit $\mathrm{B} \mid 2$ & 9 & 11 & 6 & 7 & 3 \\
\hline MGUS & 4 & 2 & 2 & 5 & 3 \\
\hline Alcoholic & 2 & 4 & 5 & 9 & I \\
\hline Immune & 3 & 4 & 2 & 6 & 3 \\
\hline Hereditary & 2 & 3 & 2 & 2 & I \\
\hline Other & 5 & 4 & 7 & 6 & 5 \\
\hline TCSS & $12.3 \pm 4.3$ & $12.0 \pm 3.8$ & $11.9 \pm 4.2$ & $13.2 \pm 3.5$ & $12.5 \pm 4.0$ \\
\hline and average dose & N/A & N/A & $\begin{array}{l}\text { Amitryptyline }(n=15) \\
17.5 \pm 12.5 \mathrm{mg} / \mathrm{d} \\
\text { Nortriptyline }(\mathrm{n}=2) \\
50 \mathrm{mg} / \mathrm{d} \\
\text { Carbamazepine }(\mathrm{n}=2) \text {, } \\
300 \pm \mathrm{I} \mid \mathrm{I} \mathrm{mg} / \mathrm{d} \\
\text { Valproic acid }(\mathrm{n}=\mathrm{I}) \\
750 \mathrm{mg} / \mathrm{d} \\
\text { Phenytoin }(\mathrm{n}=2), \\
250 \mathrm{mg} / \mathrm{d} \\
\text { Nabilone }(\mathrm{n}=3) \\
\mathrm{I.50} \pm 0.50 \mathrm{mg} / \mathrm{d} \\
\text { Morphine }(\mathrm{n}=14) \\
54 \pm 27 \mathrm{mg} / \mathrm{d} \\
\text { Fentanyl }(\mathrm{n}=1) \\
50 \mu \mathrm{Ig} / \mathrm{d} \\
\text { Oxycodone }(\mathrm{n}=7) \\
33 \pm 17 \mathrm{mg} / \mathrm{d} \\
\text { Acetaminophen }(\mathrm{n}=4) \\
722 \pm 368 \mathrm{mg} / \mathrm{d} \\
\text { Codeine }(\mathrm{n}=2) \\
105 \pm 86 \mathrm{mg} / \mathrm{d}\end{array}$ & $\begin{array}{l}\text { Phenytoin }(\mathrm{n}=2) \\
250 \pm 70 \mathrm{mg} / \mathrm{d} \\
\text { Venlafaxine }(\mathrm{n}=3) \\
150 \mathrm{mg} / \mathrm{d} \\
\text { Morphine }(\mathrm{n}=20) \\
48 \pm 23 \mathrm{mg} / \mathrm{d} \\
\text { Fentanyl }(\mathrm{n}=2) \\
63 \pm 18 \mu \mathrm{g} / \mathrm{d} \\
\text { Oxycodone }(\mathrm{n}=13 \\
39 \pm 18 \mathrm{mg} / \mathrm{d} \\
\text { Acetaminophen }(\mathrm{n}=2) \\
650 \pm 168 \mathrm{mg} / \mathrm{d} \\
\text { Codeine }(\mathrm{n}=4) \\
141 \pm 77 \mathrm{mg} / \mathrm{d}\end{array}$ & N/A \\
\hline \multicolumn{6}{|c|}{ Pre-existing side effects of $\mathrm{NeP}$ therapies } \\
\hline Sedation & & & 14 (31\%) & 17 (26\%) & \\
\hline $\begin{array}{l}\text { Dizziness } \\
\text { (lightheadedness) }\end{array}$ & & & $10(22 \%)$ & $16(25 \%)$ & \\
\hline Peripheral edema & & & I (2\%) & $\mathrm{I}(2 \%)$ & \\
\hline Fatigue & & & 14 (3l\%) & $12(19 \%)$ & \\
\hline
\end{tabular}


Table I (Continued)

\begin{tabular}{|c|c|c|c|c|c|}
\hline \multirow[t]{2}{*}{ Clinical features } & \multicolumn{2}{|c|}{ Monotherapy } & \multicolumn{3}{|c|}{ Adjuvant therapy } \\
\hline & $\begin{array}{l}\text { Venlafaxine } \\
(n=43)\end{array}$ & $\begin{array}{l}\text { Gabapentin } \\
(n=52)\end{array}$ & $\begin{array}{l}\text { Venlafaxine } \\
(n=45)\end{array}$ & $\begin{array}{l}\text { Gabapentin } \\
(n=64)\end{array}$ & $\begin{array}{l}\text { Control group } \\
\text { (no therapy) } \\
(n=29)\end{array}$ \\
\hline Dry mouth & & & $5(11 \%)$ & $5(8 \%)$ & \\
\hline Headache & & & $6(13 \%)$ & $4(6 \%)$ & \\
\hline Other & & & $15(33 \%)$ & $20(31 \%)$ & \\
\hline $\begin{array}{l}\text { Total responses of } \\
\text { adverse effects }\end{array}$ & & & 65 & 75 & \\
\hline $\begin{array}{l}\text { Number of patients } \\
\text { with adverse effects } \\
\text { prior to initiation of } \\
\text { studied therapies }\end{array}$ & & & 20 (44\%) & 25 (39\%) & \\
\hline $\begin{array}{l}\text { Duration of time using } \\
\text { NeP therapy prior to } \\
\text { initiation of studied } \\
\text { therapies (months) }\end{array}$ & & & $11.9 \pm 5.2$ & $14.6 \pm 6.1$ & \\
\hline
\end{tabular}

Notes: Data are presented as mean \pm standard deviation, or as an absolute number. ANOVA tests were performed to compare groups receiving monotherapy as well as the two groups receiving adjuvant therapy. *indicates a significant difference with ANOVA testing when the intervention group was compared to the control group.

Abbreviations: MGUS, monoclonal gammopathy of uncertain significance; NeP, neuropathic pain; PN, polyneuropathy; TCNS, Toronto Neuropathy Clinical Score.

in sleep quantity and in the sleep problems index (Table 2). Within the SF-36 domains, both venlafaxine and gabapentin monotherapy improved physical functioning, bodily pain, and vitality. Venlafaxine monotherapy further improved the SF-36 domains of general health and mental health. Both gabapentin and venlafaxine monotherapy led to improvements in BPI subscales, including average pain, present pain; as well as with pain interference with general activity, walking ability, normal work, social relations, sleep, and enjoyment of life. Venlafaxine monotherapy additionally assisted with pain related interference with mood (Table 2). Monotherapy with venlafaxine improved the total HADS score as well as the HADS-A score (but not the HADS-D score). Monotherapy with gabapentin improved the HADS-A score only (Table 2).

\section{Adjuvant therapy}

There were no significant improvements in EQ-5D scores or EQ-Health status scores at 6 month visits versus baseline for any adjuvant therapy treatment group (Table 3). However, venlafaxine adjuvant therapy was associated with improved EQ-5D Self Care scoring at 6 months. Both gabapentin and venlafaxine adjuvant therapy was associated with improvement in sleep disturbance within the MOSSS (Table 3). Venlafaxine adjuvant therapy was further associated with additional improvements in sleep adequacy, sleep quantity and in the sleep problems index (Table 3). Within the SF-36 domains, both venlafaxine and gabapentin adjuvant therapy improved physical functioning and bodily pain. Venlafaxine adjuvant therapy further improved the SF-36 domain of mental health. Both gabapentin and venlafaxine adjuvant therapy improved
BPI subscales including average pain and interference with mood and sleep. Venlafaxine adjuvant therapy additionally assisted with pain-related interference for walking ability and social relations, while gabapentin adjuvant therapy additionally assisted with pain-related interference for general activity, normal work, and enjoyment of life (Table 3). Adjuvant therapy with venlafaxine improved the total HADS score as well as the HADS-A score (but not the HADS-D score); gabapentin adjuvant therapy improved the HADS-A score only (Table 2).

\section{Adverse events}

All treatment groups suffered some attrition. Discontinuation rates ranged from $19 \%$ to $46 \%$ in each group, the greatest percentage of discontinuation being seen in the gabapentin monotherapy group. Discontinuations were related to development of intolerable side effects as well as drug inefficacy. Although gabapentin therapy had a trend towards greater discontinuation of pharmacological therapy, there were no significant differences between intervention groups.

In the monotherapy groups, a total of 21 patients stopped treatment at or before the 3-month follow-up visit. The most common side effects for all patients receiving either monotherapy were sedation, dizziness/lightheadedness and fatigue. At the 6-month visits, an additional 8 patients discontinued their medication due to inefficacy rather than intolerable side effects.

In the adjuvant therapy groups, a total of 17 patients discontinued medication at 3-month follow-up visits, due to a combination of intolerable side effects and perceived inefficacy. Sedation was found to be the most common side 


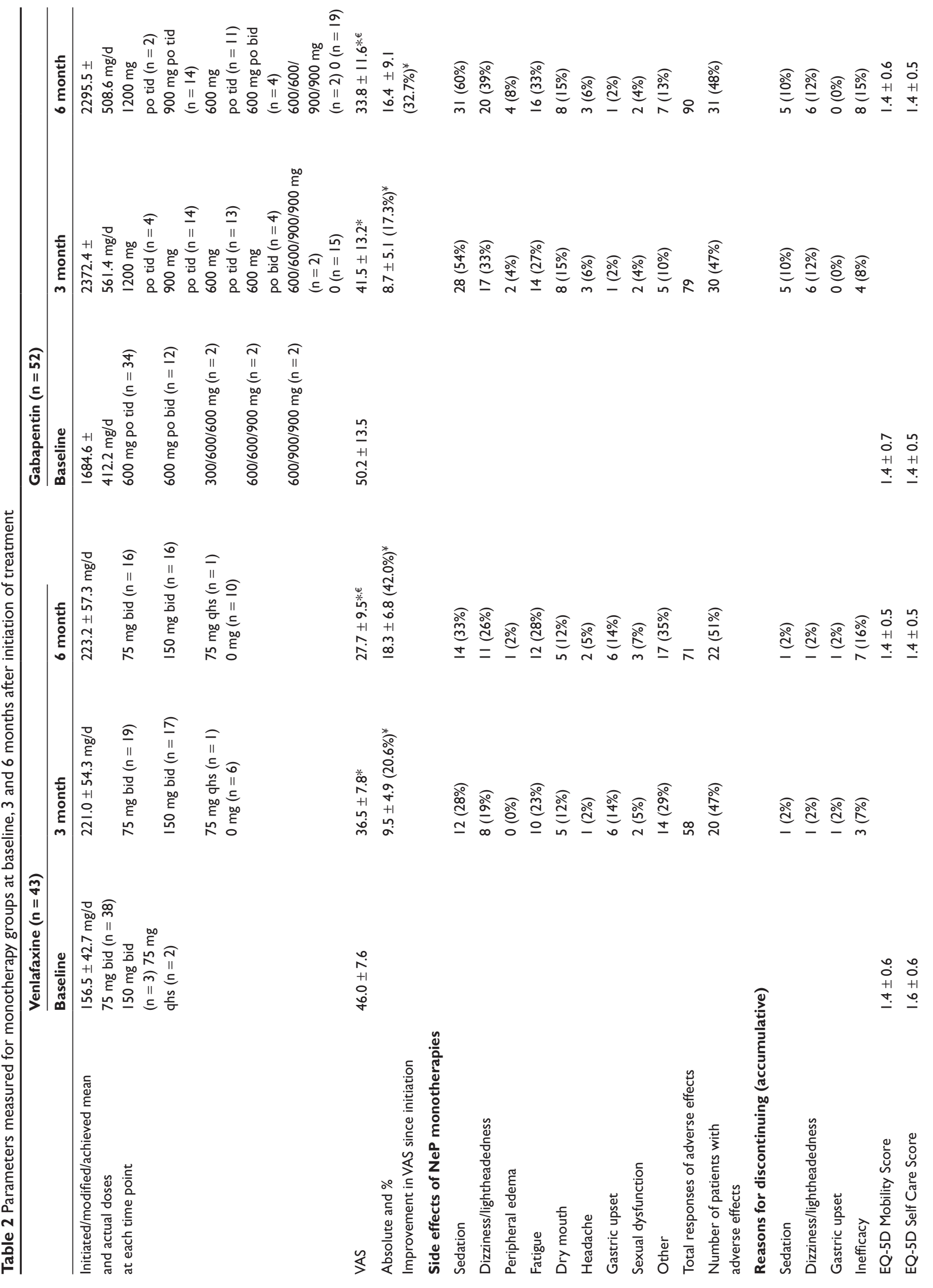




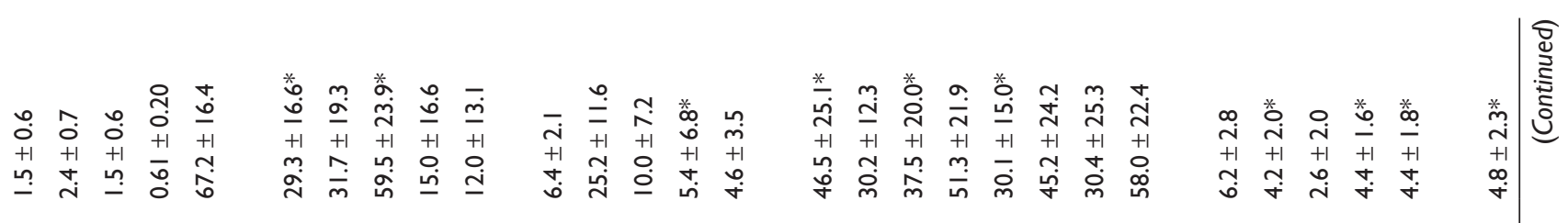

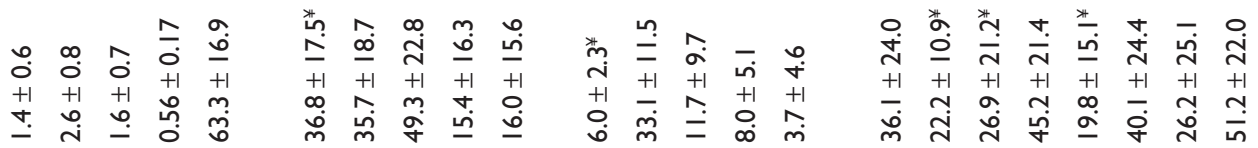

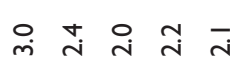

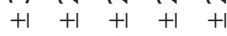

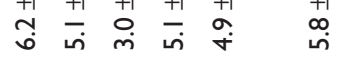

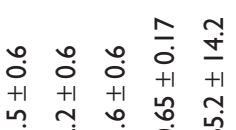

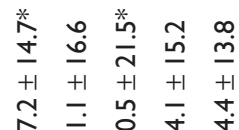

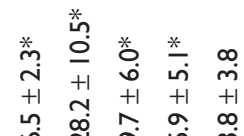

茫

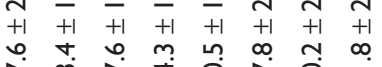

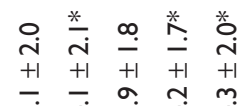

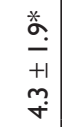

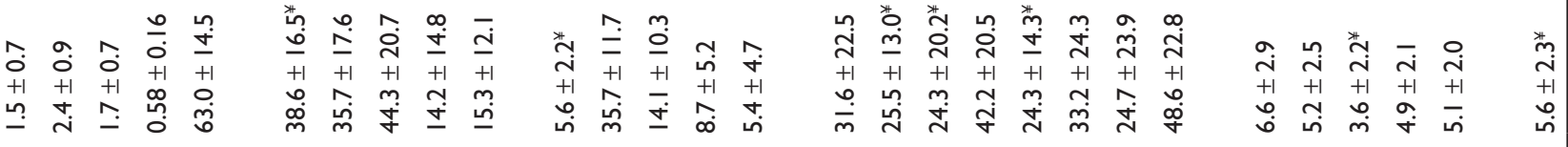
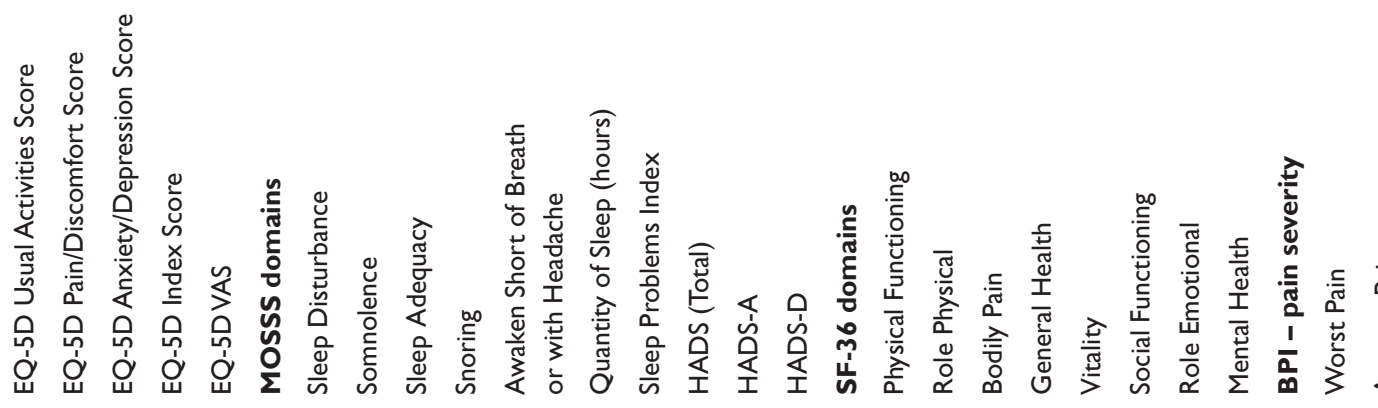


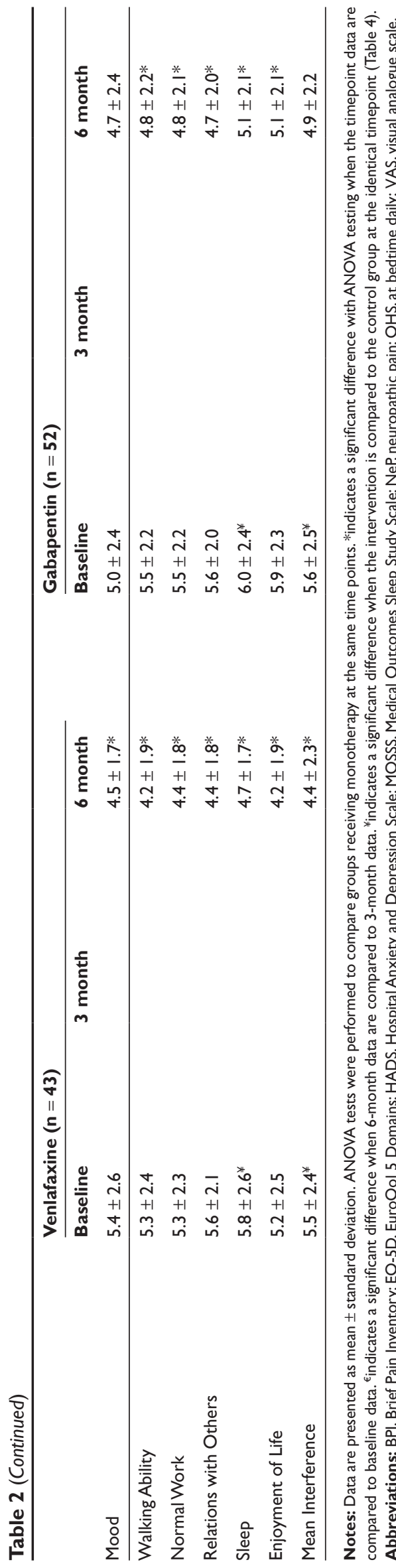

effect in both adjuvant treatment groups. An additional 8 patients receiving adjuvant therapy discontinued treatment at or before the 6-month follow-up visit due to medication inefficacy. There were no serious adverse events occurring within any treatment group.

\section{Patient assessment}

Global improvement was evaluated with the PGIC. On both the clinician-rated and the patient-rated instruments, there were responses in favor of monotherapy with either venlafaxine or gabapentin compared to the control group (no therapy), whereas the adjuvant therapies were associated with less beneficial but significant responses (Figure 2).

\section{Control group (no therapy)}

The control group was stable over time in their levels of pain, sleep parameters, mood and anxiety scale values and in quality and functioning of life parameters (Table 4). None of these patients started other forms of therapy over the 6-month assessment period.

\section{Discussion}

Although there are guidelines for the management of NeP, very few head-to-head comparisons of pharmacotherapies exist. The present study suggests that venlafaxine as monotherapy or adjuvant treatment for $\mathrm{NeP}$ has similar benefits on pain severity, sleep, anxiety/depression and functioning compared to gabapentin. Based on our open-label results, venlafaxine adjuvant or monotherapy should be considered in NeP patients. Modulation of multiple NeP pathogenic pathways ("rational polytherapy") may be beneficial in numerous patients with $\mathrm{NeP}$, as demonstrated by the efficacy of adjuvant therapy with either therapy of interest in this study.

We decided to use gabapentin as a comparator given its widespread use, reasonable adverse event profile, therapeutic benefits at sub-maximal dosing (1800 mg/day), low cost and acceptance as beneficial NeP therapy. ${ }^{13,18,36} \mathrm{It}$ was expected that all treatment groups would fare better than the untreated, or control, group. Although the control group had lower VAS pain scores that changed little over time, there were similar improvements for both therapy groups that were not witnessed in the control group. Both venlafaxine and gabapentin, with proven efficacy in the treatment of $\mathrm{NeP},{ }^{12,23,37}$ demonstrated improvement in VAS pain scores, but also were associated with some improvements in sleep, pain-associated psychiatric difficulties, and functional abilities. Previous studies examining low dose venlafaxine asserted a number needed to treat (NNT) 


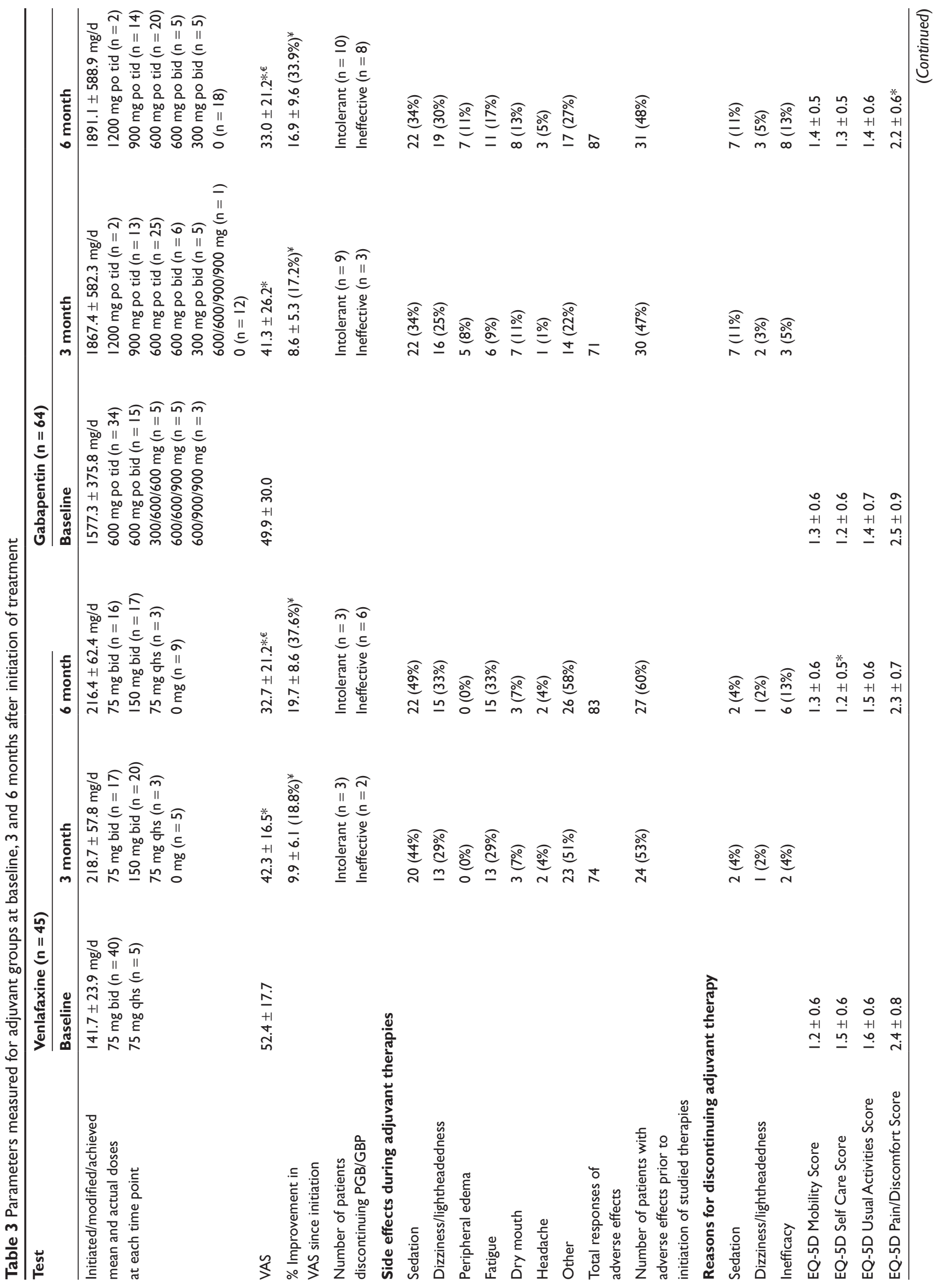




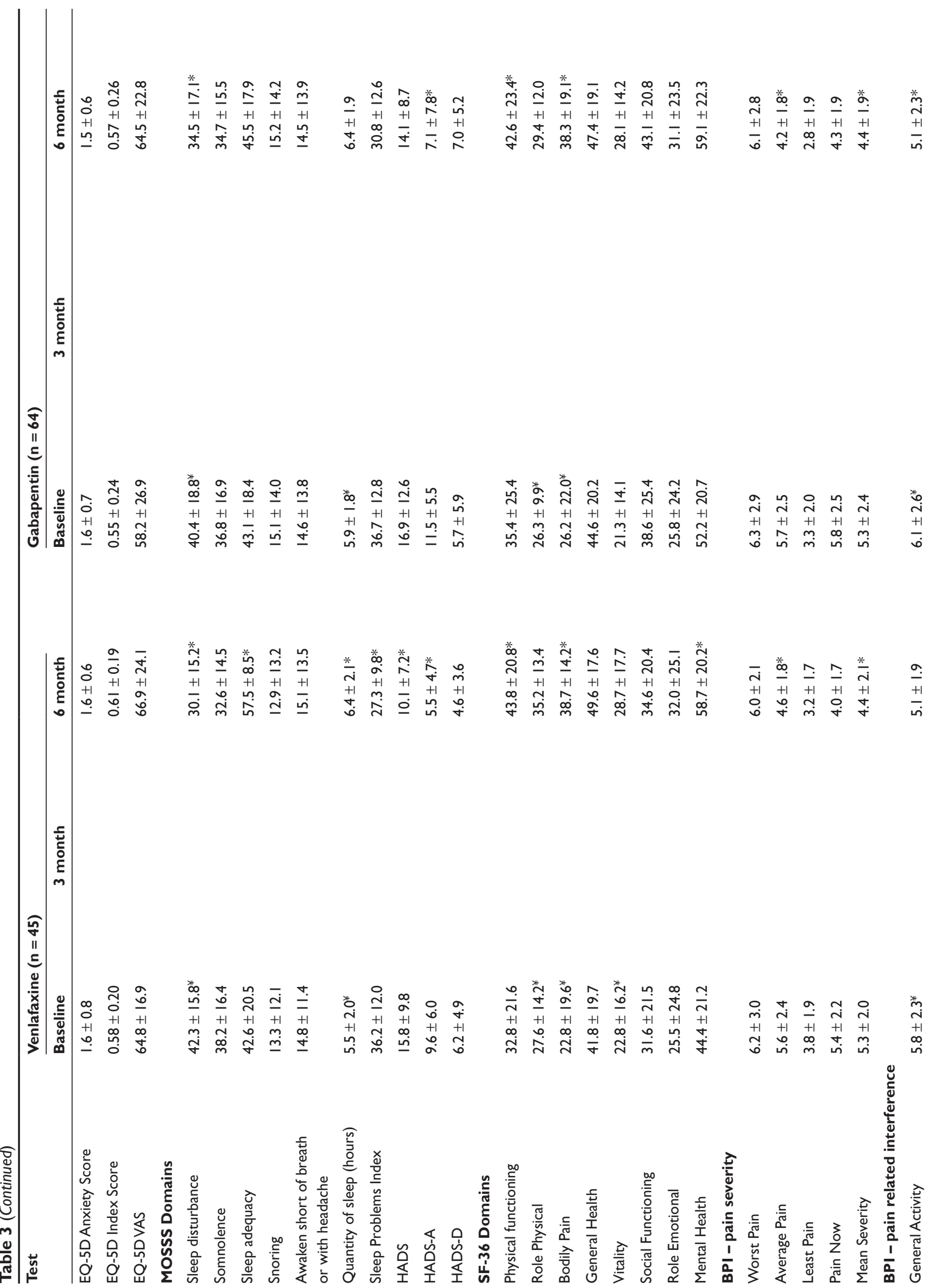




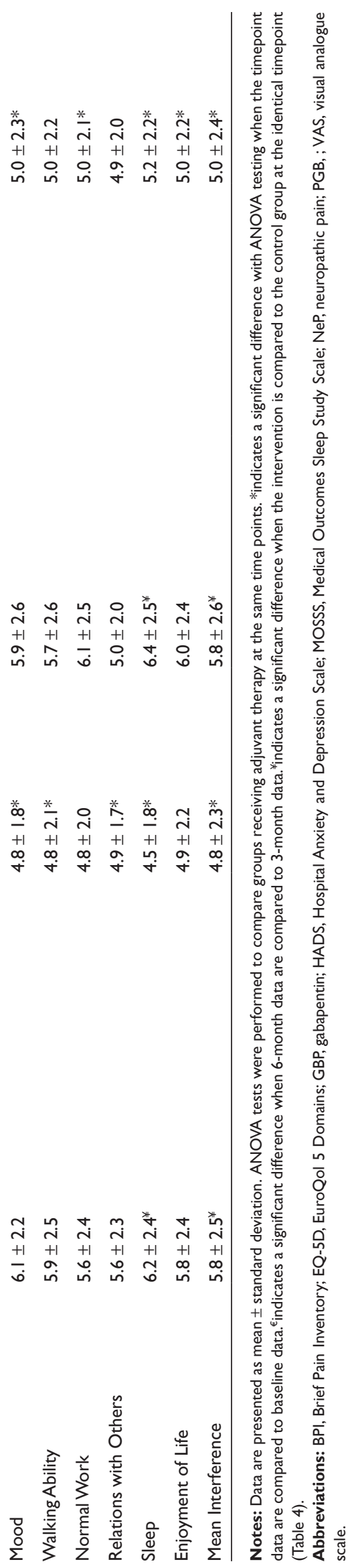

of 5.5 (3.4-13.5), ${ }^{22}$ while higher dosing of venlafaxine provides a NNT of $4.6(2.9-10.6)^{22}-$ thus, venlafaxine is suggested to be provided at a minimum of $\geq 150 \mathrm{mg}$ daily ${ }^{23}$ in order to manage pain. In comparison, gabapentin for management of $\mathrm{NeP}$ due to a peripheral nervous system disease is associated with an NNT of $4.3(2.8-8.6) .{ }^{12}$ Therefore, the expected therapeutic benefit for both groups is similar. In our study, the two pharmacotherapies were quite comparable for most parameters, although venlafaxine had possibly better efficacy in management of anxiety/ depression and sleep dysfunction than gabapentin. Our results suggest that venlafaxine is also an effective medication in NeP therapy, with benefits that are comparable to those of gabapentin.

The most common adverse event with the use of either venlafaxine or gabapentin was sedation, although the numbers of adverse events did not differ between the two therapies assessed. Sedation, lightheadedness/dizziness and fatigue were the most common adverse events to lead to discontinuation of therapy in all intervention groups. Inefficacy also occurred in $13 \%$ to $16 \%$ of patients with either pharmacotherapy. Global benefit assessed with the PGIC found overall beneficial effects within any of the four intervention groups compared to that of the control group receiving no pharmacotherapy.

Another SNRI, duloxetine, also reduces pain in patients with major depressive disorder. ${ }^{38}$ Interestingly, a post-hoc analysis of two independent, randomized, controlled trials in patients with major depressive disorder comparing duloxetine with placebo identified that approximately $50 \%$ of duloxetine's total effect on overall pain was independent of responses in depression, suggesting an independent analgesic effect of duloxetine which may contribute to efficacy in diabetic peripheral $\mathrm{NeP}^{20,21}$ and fibromyalgia. ${ }^{39-41}$ The reuptake inhibition of both serotonin and norepinephrine has remained the proposed mechanism by which SNRIs alleviates pain and improves mood through increased availability of serotonin and norepinephrine, important neurotransmitters in descending pain inhibitory pathways in the central nervous system. ${ }^{42}$

There are a number of limitations associated with our results. The greatest limitation was a lack of randomization and blinding. The flexible dosing and variability in overall dosing performed was analogous to everyday clinical therapy, but may certainly limit the direct comparison of the agents considered. Selection bias may have occurred based upon the physician and patient choosing the desired therapy - in particular, it is possible, but unintended, that 
patients with history of psychiatric illness may have been more likely to start on venlafaxine. Flexible dosing, although commonly used in routine management, may have contributed to variability in efficacy or adverse events. There is no optimal control group for comparison - we selected a cohort group who chose to receive no pharmacotherapy, but these patients may have expectations of no improvement over time, and had lower baseline VAS scores. It is extremely difficult to control for such variables without performing a randomized, double-blinded, controlled study, although the expense of such a study with an assumed very large sample size is likely prohibitive. Although all patients were encouraged to use conservative measure to assist with $\mathrm{NeP}$ relief, including aerobic forms of exercise, there was no means of controlling for non-pharmaceutical interventions, nor was there any means of controlling for patients who also used over-the-counter medications for pain relief. Patients referred to our tertiary care clinic may have not been representative of the general population of patients with PN and PN-mediated NeP.
A

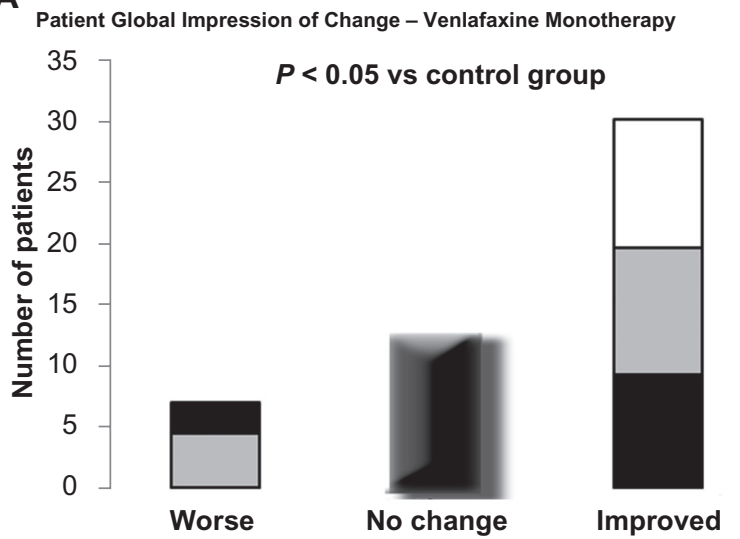

C

Patient Global Impression of Change - Venlafaxine Adjuvant Therapy

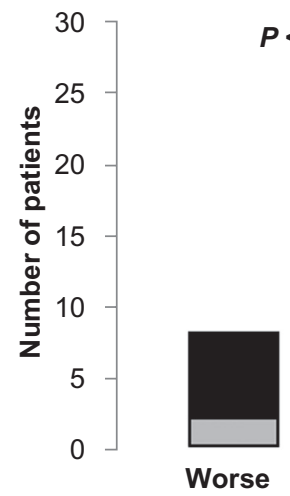

E

Patient Global Impression of Change - Control Group (No Therapy)

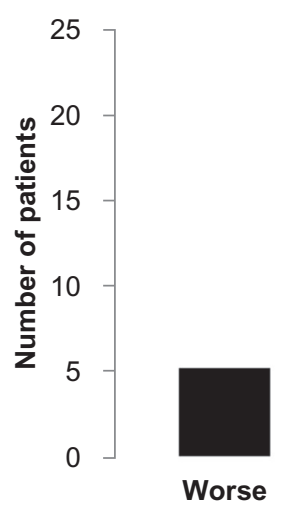

$P<0.05$ vs control group

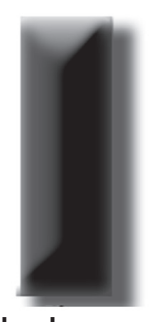

No change

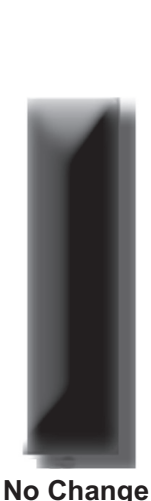

B

Patient Global Impression of Change - Gabapentin Monotherapy

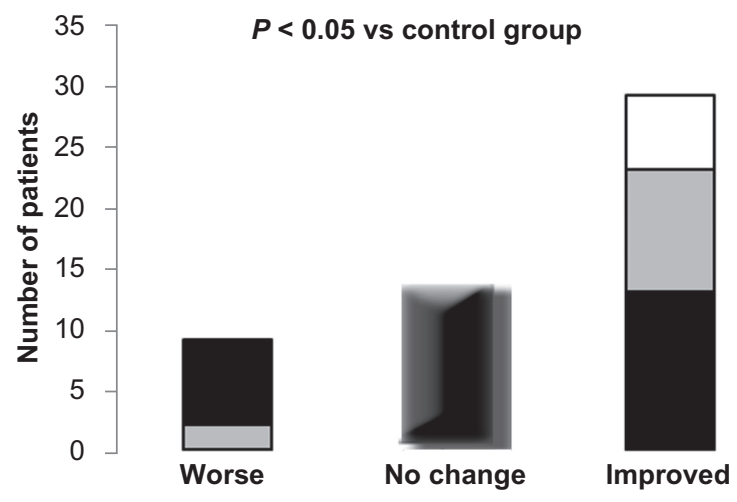

D

Patient Global Impression of Change - Gabapentin Adjuvant Therapy

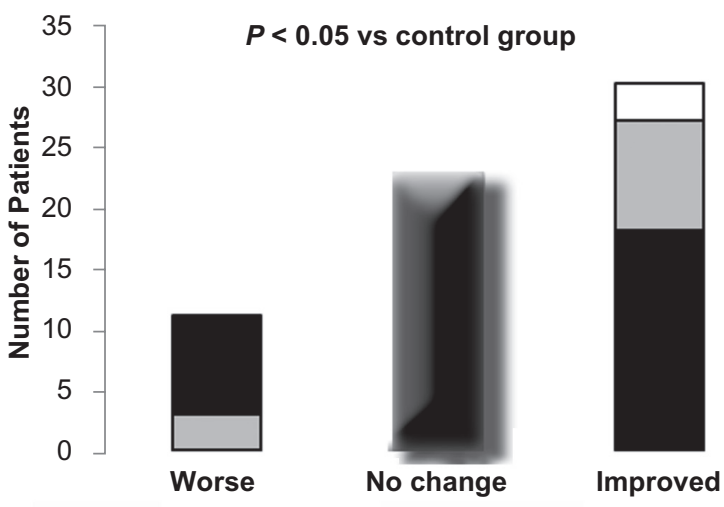

Figure 2 Patient global impression of change (PGIC) was analyzed using a Cochran-Mantel-Haenszel procedure, adjusting for center in each case. Patients reported a significant perceived benefit with monotherapy compared to control group patients for each of venlafaxine (A) and gabapentin (B), as well as with adjuvant therapy for each of venlafaxine (C) and gabapentin (D). In contrast, the control group receiving no therapy had no significant change in PGIC reported (E). 
Table 4 Parameters measured for the control groups at baseline, 3 and 6 months after initiation of treatment

\begin{tabular}{|c|c|c|c|}
\hline & \multicolumn{3}{|c|}{ Control group $(n=29)$} \\
\hline & Baseline & 3 month & 6 month \\
\hline VAS & $29.6 \pm 12.1$ & $30.3 \pm 12.7$ & $30.4 \pm 1.5$ \\
\hline Absolute and \% & & $-0.7 \pm 6.8(-2.3 \%)$ & $-0.8 \pm 7.2(-2.7 \%)$ \\
\hline \multicolumn{4}{|l|}{ Improvement in VAS since initiation } \\
\hline EQ-5D Mobility Score & $1.3 \pm 0.8$ & & $1.3 \pm 0.8$ \\
\hline EQ-5D Self Care Score & $1.2 \pm 0.8$ & & $1.3 \pm 0.7$ \\
\hline EQ-5D Usual Activities Score & $1.3 \pm 1.0$ & & $1.3 \pm 0.9$ \\
\hline EQ-5D Pain/Discomfort Score & $2.1 \pm 0.9$ & & $2.1 \pm 0.9$ \\
\hline EQ-5D Anxiety/Depression Score & $1.6 \pm 0.9$ & & $1.6 \pm 0.8$ \\
\hline EQ-5D Index Score & $0.68 \pm 0.26$ & & $0.67 \pm 0.28$ \\
\hline EQ-5DVAS & $69.8 \pm 21.5$ & & $69.3 \pm 21.2$ \\
\hline \multicolumn{4}{|l|}{ MOsss Domains } \\
\hline Sleep Disturbance & $28.4 \pm 21.2$ & & $28.8 \pm 22.0$ \\
\hline Somnolence & $27.3 \pm 21.5$ & & $28.4 \pm 22.5$ \\
\hline Sleep Adequacy & $56.4 \pm 27.2$ & & $55.8 \pm 26.8$ \\
\hline Snoring & $13.7 \pm 16.6$ & & $14.0 \pm 14.6$ \\
\hline Awaken Short of Breath or with Headache & $12.4 \pm 17.1$ & & $14.3 \pm 19.2$ \\
\hline Quantity of Sleep (hours) & $7.2 \pm 2.8$ & & $7.1 \pm 2.9$ \\
\hline Sleep Problems Index & $23.6 \pm 16.7$ & & $24.1 \pm 18.2$ \\
\hline HADS (total) & $9.4 \pm 10.2$ & & $9.2 \pm 10.8$ \\
\hline HADS-A & $5.3 \pm 11.6$ & & $5.1 \pm 11.8$ \\
\hline HADS-D & $4.1 \pm 11.9$ & & $4.1 \pm 11.6$ \\
\hline \multicolumn{4}{|l|}{ SF-36 Domains } \\
\hline Physical Functioning & $48.9 \pm 31.3$ & & $49.7 \pm 32.0$ \\
\hline Role Physical & $36.5 \pm 17.8$ & & $35.1 \pm 16.5$ \\
\hline Bodily Pain & $40.2 \pm 19.2$ & & $42.6 \pm 15.2$ \\
\hline General Health & $59.7 \pm 24.7$ & & $54.4 \pm 23.8$ \\
\hline Vitality & $37.4 \pm 23.5$ & & $39.0 \pm 24.1$ \\
\hline Social Functioning & $51.2 \pm 28.3$ & & $54.7 \pm 26.2$ \\
\hline Role Emotional & $40.6 \pm 29.9$ & & $4 I . I \pm 28.0$ \\
\hline Mental Health & $63.7 \pm 27.1$ & & $62.5 \pm 24.6$ \\
\hline \multicolumn{4}{|l|}{ BPI - pain severity } \\
\hline Worst Pain & $5.4 \pm 3.5$ & & $5.7 \pm 3.4$ \\
\hline Average Pain & $4.1 \pm 3.3$ & & $4.2 \pm 3.2$ \\
\hline Least Pain & $1.5 \pm 2.9$ & & $1.6 \pm 3.1$ \\
\hline Pain Now & $4.7 \pm 3.2$ & & $4.5 \pm 3.0$ \\
\hline Mean Severity & $3.9 \pm 3.1$ & & $4.0 \pm 3.2$ \\
\hline \multicolumn{4}{|l|}{ BPI - pain related interference } \\
\hline General Activity & $4.1 \pm 3.1$ & & $4.0 \pm 3.1$ \\
\hline Mood & $3.2 \pm 3.2$ & & $3.4 \pm 3.1$ \\
\hline Walking Ability & $3.5 \pm 3.0$ & & $3.7 \pm 2.8$ \\
\hline Normal Work & $3.0 \pm 3.1$ & & $3.0 \pm 3.0$ \\
\hline Relations with Others & $3.2 \pm 2.7$ & & $3.0 \pm 2.8$ \\
\hline Sleep & $2.6 \pm 3.1$ & & $2.5 \pm 3.0$ \\
\hline Enjoyment of Life & $4.1 \pm 2.8$ & & $3.9 \pm 2.7$ \\
\hline Mean Interference & $3.4 \pm 3.0$ & & $3.4 \pm 3.1$ \\
\hline
\end{tabular}

Notes: Data are presented as mean \pm standard deviation. ANOVA tests were performed to compare groups receiving monotherapy at the same time points.

Abbreviations: BPI, Brief Pain Inventory; EQ-5D, EuroQol 5 Domains; HADS, Hospital Anxiety and Depression Scale; MOSSS, Medical Outcomes Sleep Study Scale; NeP, neuropathic pain; VAS, visual analogue scale. 
$\mathrm{NeP}$ is a significant debilitating sequelae of PN that usually demands pharmacotheapy. We advocate for future randomized, blinded, head-to-head studies of pharmacotherapies in the management of NeP. Future studies examining combination therapy using agents with distinct mechanisms of action, such as venlafaxine and gabapentin, will be of benefit to determine possible additive effects. Our results suggest that therapies for $\mathrm{NeP}$ modulate parameters of sleep and mood/anxiety, and enhance functional abilities in addition to modifying pain severity. We suggest that both venlafaxine and gabapentin are appropriate and comparable adjuvant therapies and monotherapies in the management of $\mathrm{NeP}$.

\section{Disclosures}

The authors have no financial interests to disclose.

\section{References}

1. Hughes RA. Peripheral neuropathy. BMJ. 2002;324(7335):466-469.

2. Martyn CN, Hughes RA. Epidemiology of peripheral neuropathy. J Neurol Neurosurg Psychiatry. 1997;62(4):310-318.

3. Dyck PJ, Oviatt KF, Lambert EH. Intensive evaluation of referred unclassified neuropathies yields improved diagnosis. Ann Neurol. 1981;10(3):222-226.

4. Toth C, Au S. A prospective identification of neuropathic pain in specific chronic polyneuropathy syndromes and response to pharmacological therapy. Pain. 2008;138(3):657-666.

5. Backonja M, Beydoun A, Edwards KR, et al. Gabapentin for the symptomatic treatment of painful neuropathy in patients with diabetes mellitus: a randomized controlled trial. JAMA. 1998;280(21):1831-1836.

6. Gordon A, Choiniere M, Collet JP. The humanistic burden of neuropathic pain in Canada. Journal of Outcomes Research. 2006; 2006:23-35.

7. Mauskopf J, Austin R, Dix L, Berzon R. The Nottingham Health Profile as a measure of quality of life in zoster patients: convergent and discriminant validity. Qual Life Res. 1994;3(6):431-435.

8. McDermott AM, Toelle TR, Rowbotham DJ, et al. The burden of neuropathic pain: results from a cross-sectional survey. Eur J Pain. 2006;10(2):127-135.

9. Meyer-Rosberg K, Burckhardt CS, Huizar K, et al. A comparison of the SF-36 and Nottingham Health Profile in patients with chronic neuropathic pain. Eur J Pain. 2001;5(4):391-403.

10. Bennett MI, Smith BH, Torrance N, Potter J. The S-LANSS score for identifying pain of predominantly neuropathic origin: validation for use in clinical and postal research. J Pain. 2005;6(3):149-158.

11. Bouhassira D, Attal N, Alchaar H, et al. Comparison of pain syndromes associated with nervous or somatic lesions and development of a new neuropathic pain diagnostic questionnaire(DN4). Pain. 2005; 114(1-2):29-36.

12. Sindrup SH, Otto M, Finnerup NB, Jensen TS. Antidepressants in the treatment of neuropathic pain. Basic Clin Pharmacol Toxicol. 2005;96(6):399-409.

13. Moulin DE, Clark AJ, Gilron I, et al. Pharmacological management of chronic neuropathic pain - consensus statement and guidelines from the Canadian Pain Society. Pain Res Manag. 2007;12(1):13-21.

14. Chong MS, Hester J. Diabetic painful neuropathy: current and future treatment options. Drugs. 2007;67(4):569-585.

15. Gilron I, Watson CP, Cahill CM, Moulin DE. Neuropathic pain: a practical guide for the clinician. CMAJ. 2006;175(3):265-275.
16. Backonja MM. Gabapentin monotherapy for the symptomatic treatment of painful neuropathy: a multicenter, double-blind, placebo-controlled trial in patients with diabetes mellitus. Epilepsia. 1999;40 Suppl 6: S57-S59.

17. Dallocchio C, Buffa C, Mazzarello P, Chiroli S. Gabapentin vs amitriptyline in painful diabetic neuropathy: an open-label pilot study. J Pain Symptom Manage. 2000;20(4):280-285.

18. Gilron I, Bailey JM, Tu D, et al. Morphine, gabapentin, or their combination for neuropathic pain. N Engl J Med. 2005;352(13):1324-1334.

19. Sindrup SH, Jensen TS. Efficacy of pharmacological treatments of neuropathic pain: an update and effect related to mechanism of drug action. Pain. 1999;83(3):389-400.

20. Goldstein DJ, Lu Y, Detke MJ, et al. Duloxetine vs. placebo in patients with painful diabetic neuropathy. Pain. 2005;116(1-2):109-118.

21. Raskin J, Pritchett YL, Wang F, et al. A double-blind, randomized multicenter trial comparing duloxetine with placebo in the management of diabetic peripheral neuropathic pain. Pain Med. 2005;6(5):346-356.

22. Sindrup SH, Bach FW, Madsen C, et al. Venlafaxine versus imipramine in painful polyneuropathy: a randomized, controlled trial. Neurology. 2003;60(8):1284-1289.

23. Rowbotham MC, Goli V, Kunz NR, Lei D. Venlafaxine extended release in the treatment of painful diabetic neuropathy: a double-blind, placebo-controlled study. Pain. 2004;110(3):697-706.

24. Khalifa M, Daleau P, Turgeon J. Mechanism of sodium channel block by venlafaxine in guinea pig ventricular myocytes. $J$ Pharmacol Exp Ther. 1999;291(1):280-284.

25. Sumpton JE, Moulin DE. Treatment of neuropathic pain with venlafaxine. Ann Pharmacother. 2001;35(5):557-559.

26. Simpson DA. Gabapentin and venlafaxine for the treatment of painful diabetic neuropathy. J Clin Neuromuscul Dis. 2001;3(2):53-62.

27. Muth EA, Moyer JA, Haskins JT, et al. Biochemical, neurophysiological, and behavioral effects of Wy-45,233 and other identified metabolites of the antidepressant venlafaxine. Drug Dev Res. 2004;23:191-199.

28. Bril V, Perkins BA. Validation of the Toronto Clinical Scoring System for diabetic polyneuropathy. Diabetes Care. 2002;25(11): 2048-2052.

29. Perkins BA, Olaleye D, Zinman B, Bril V. Simple screening tests for peripheral neuropathy in the diabetes clinic. Diabetes Care. 2001;24(2):250-256.

30. England JD, Gronseth GS, Franklin G, et al. Evaluation of distal symmetric polyneuropathy: the role of laboratory and genetic testing (an evidence-based review). Muscle Nerve. 2009;39(1):116-125.

31. England JD, Gronseth GS, Franklin G, et al. Distal symmetric polyneuropathy: a definition for clinical research: report of the American Academy of Neurology, the American Association of Electrodiagnostic Medicine, and the American Academy of Physical Medicine and Rehabilitation. Neurology. 2005;64(2):199-207.

32. Toth $\mathrm{C}$, Lander J, Wiebe $\mathrm{S}$. The prevalance and impact of chronic pain with neuropathic pain symptoms in the general population. Pain Med. 2009;10(5):918-929.

33. Kind P, Dolan P, Gudex C, Williams A. Variations in population health stuatus: results from a United Kingdom national questionnaire survey. BMJ. 1998;316:736-741.

34. Hays RD, Stewart AL. Sleep measures. In: Stewart AL, Ware JE, editors. Measuring Functioning and Well-being: The Medical Outcomes Study Approach. Durham, NC: Duke University Press; 1992. p. 235-259.

35. Zigmond AS, Snaith RP. The hospital anxiety and depression scale. Acta Psychiatr Scand. 1983;67(6):361-370.

36. Attal N, Cruccu G, Haanpaa M, et al. EFNS guidelines on pharmacological treatment of neuropathic pain. Eur J Neurol. 2006;13(11): $1153-1169$

37. Backonja M, Beydoun A, Edwards KR, et al. Gabapentin for the symptomatic treatment of painful neuropathy in patients with diabetes mellitus: a randomized controlled trial. JAMA. 1998;280(21):1831-1836.

38. Goldstein DJ, Lu Y, Detke MJ, et al. Effects of duloxetine on painful physical symptoms associated with depression. Psychosomatics. 2004;45(1):17-28. 
39. Arnold LM, Lu Y, Crofford LJ, et al. A double-blind, multicenter trial comparing duloxetine with placebo in the treatment of fibromyalgia patients with or without major depressive disorder. Arthritis Rheum. 2004;50(9):2974-2984.

40. Arnold LM, Rosen A, Pritchett YL, et al. A randomized, double-blind, placebo-controlled trial of duloxetine in the treatment of women with fibromyalgia with or without major depressive disorder. Pain. 2005;119(1-3):5-15.
41. Russell IJ, Mease PJ, Smith TR, et al. Efficacy and safety of duloxetine for treatment of fibromyalgia in patients with or without major depressive disorder: Results from a 6-month, randomized, double-blind, placebo-controlled, fixed-dose trial. Pain. 2008;136(3): 432-444.

42. Millan MJ. Descending control of pain. Prog Neurobiol. 2002;66(6): $355-474$.
Journal of Pain Research

\section{Publish your work in this journal}

The Journal of Pain Research is an international, peer-reviewed, open access, online journal that welcomes laboratory and clinical findings in the fields of pain research and the prevention and managemen of pain. Original research, reviews, symposium reports, hypothesis formation and commentaries are all considered for publication.
The manuscript management system is completely online and includes a very quick and fair peer-review system, which is all easy to use. Visit http://www.dovepress.com/testimonials.php to read real quotes from published authors.

\footnotetext{
Submit your manuscript here: http://www.dovepress.com/journal-of-pain-research-journal
} 